\title{
Participatory Design with Blind Users: A Scenario-Based Approach
}

\author{
Nuzhah Gooda Sahib, Tony Stockman, Anastasios Tombros, and Oussama Metatla \\ School of Electronic Engineering and Computer Science, Queen Mary University of London, \\ Mile End Road, London E1 4NS, United Kingdom \\ \{nuzhah, tony.stockman, tassos, oussama\} deecs.qmul .ac.uk
}

\begin{abstract}
Through out the design process, designers have to consider the needs of potential users. This is particularly important, but rather harder, when the designers interact with the artefact to-be-designed using different senses or devices than the users, for example, when sighted designers are designing an artefact for use by blind users. In such cases, designers have to ensure that the methods used to engage users in the design process and to communicate design ideas are accessible. In this paper, we describe a participatory approach with blind users based on the use of a scenario and the use of dialogue-simulated interaction during the development of a search interface. We achieved user engagement in two ways: firstly, we involved a blind user with knowledge of assistive technologies in the design team and secondly, we used a scenario as the basis of a dialogue between the designers and blind users to simulate interaction with the proposed search interface. Through this approach, we were able to verify requirements for the proposed search interface and blind searchers were able to provide formative feedback, to critique design plans and to propose new design ideas based on their experience and expertise with assistive technologies. In this paper, we describe the proposed scenario-based approach and examine the types of feedback gathered from its evaluation with blind users. We also critically reflect on the benefits and limitations of the approach, and discuss practical considerations in its application.
\end{abstract}

Keywords: scenario, participatory design, visually impaired users.

\section{$1 \quad$ Introduction}

When designing accessible interfaces, it is crucial for the designers to make sure that their understanding of the problem is aligned to the users' experience of their interactions with the interface. When designers interact with systems using different senses, devices and interface widgets than to the target population, it can be difficult for them to exclusively depend on their expertise to correctly imagine the needs of the users and to conceptualise the users' interactions with the system. Thus, designers have to be particularly sensitive as to how the users perceive technology [1].

As a result, design paradigms such as "Inclusive Design" [2][3], "Design for All" [4] and "User Sensitive Inclusive Design" [5] have been proposed to encourage 
designers to include non-standard populations such as older and disabled users in the design process. These approaches aim to give an effective voice to users in the design process and enable designers to develop real empathy towards users to ensure they communicate design ideas in an accessible form.

In this paper, we propose the use of scenarios for participatory design with blind users. We use a scenario, expressed as a textual narrative, as a basis for dialogue between designers and users in the design of a search interface. There are two levels to our approach to participatory design: firstly, we include a blind user with knowledge of assistive technologies as a full member of the design team and secondly we use the scenario and a dialogue-based interaction to gather formative feedback from 4 blind users.

The details of the finished search interface and its evaluation are reported in [6] and are not detailed in this paper. Instead, we address the question of how to successfully engage blind users in design, given that the majority of tools used for early stage prototyping by developers, such as wireframes and paper prototypes, contain barriers to participation by blind users. We focus on describing the steps in creating the scenario and its evaluation as a means of engaging blind users in the design process. Therefore, the contributions of this paper are three-fold:

(i) We propose a participatory approach based on a textual narrative scenario and a dialogue-based interaction to engage blind users in the design process.

(ii) We evaluate our approach with blind users and describe the types of feedback that we gathered.

(iii) We reflect on our approach outlining its benefits, challenges and the practical experiences that we gained from applying it so that the approach can be reused or further developed.

The rest of this paper is structured as follows: in Section 2, we describe the use of scenarios in user-centred design and discuss approaches for engaging blind users in the design process. In Section 3, we outline the steps involved in developing the scenario-based approach and we discuss the user evaluation of the proposed approach in Section 4. We reflect on the approach in Section 5, outlining the benefits, challenges and practical experiences.

\section{Related Work}

To the best of our knowledge, in the context of interface design, there is no reported research on the use of scenarios for participatory design with blind users. Thus, in this section, we discuss how scenarios have been used in usability engineering and we describe approaches to participatory design with blind users.

\subsection{Using Scenarios in Design}

The use of scenarios in the early stages of the design cycle involves designers using a description of people (actors) and their activities (tasks) to help potential users to 
envision an interface that will be developed in the future [7, p. 46]. Scenarios consist of a plot, including a sequence of actions and events, which help to emphasise and explore the goals that a user might adopt and pursue.

Scenarios enable rapid communication among different stakeholders and thus, scenario-based design approaches are iterative and lightweight for envisioning future use possibilities [8]. As a result, designers can work through ideas rapidly, obtaining feedback and refining their ideas to make quick progress. Scenarios focus the design efforts on use, that is, what people will use the interface for and how they will use it [9]. This compels designers to maintain a consideration for people and their needs, as opposed to focusing only on the technology.

Apart from their use in framing the design rationale, scenarios have been used in HCI for other purposes, namely, for planning and evaluating test tasks and to specify usability goals. In [10], Bodker highlighted how scenarios can be used at different times with different purposes and described three ways of using scenarios in usability work, namely to generate ideas during field studies, as a starting point in design workshops and for usability testing of prototypes. Scenarios also have a natural and inherent ability to support participatory design as they allow users to identify themselves as the actors in the scenario and to reflect on their own ideas and their implications in the context of design. In this way, scenario-based approaches provide a common language for discussions among users and designers [9].

Newell and McGregor [11] suggested a story-telling approach with older and disabled users to gather information and data about accessibility issues by using scenarios in the narrative form. In this respect, [12] used scenario-based drama to elicit user requirements in the design of a fall detector for elderly people. Four scenarios were developed which were performed by a theatre group and filmed. These videos were then used to engage elderly people in the design process by provoking discussions about the use of the system. Other examples of the use of scenarios to engage users in the design process are: [13] used scenarios to understand user requirements in the design of a location-based feedback notification system for users with mobility impairments and in the design of digital technologies for older users, [14] used video prompts of a scenario about the problem domain for participatory design with users who were in the $65+$ age group.

\subsection{Participatory Design with Blind Users}

Participatory design with blind users can be challenging as designers have to ensure their methods of communicating design ideas with users are appropriate and effective to gather useful feedback. In [15], Okamoto reported about a workshop where scenarios were used by visually impaired and sighted students to discuss products being designed to enhance day-to-day activities for visually impaired users. However, no details of the implementation of the scenario-based method were given, so it is difficult to understand how scenarios were used in that context. In another setting, [16] conducted a workshop including round table discussions and demonstrations of early prototypes to engage visually impaired users in the design of a system to represent diagrams in sound. 
Prototyping is also a common way of brainstorming design ideas with users, but for obvious reasons, visual prototyping techniques are not appropriate for blind users and therefore, alternatives have been proposed: [17] describes haptic paper prototypes (using cardboard mockups) while a tactile paper prototyping approach (with Braille and tactile graphics mockups) was discussed in [18]. Also, [19] proposed 2 types of haptic mock-ups for visually impaired children consisting of cardboard models and Braille-labelled plastic artefacts.

However, these techniques are time consuming to set up and changes are not easy to make in response to feedback. Both methods proposed in [18] and [19] exclude the significant proportion of the blind population who are not Braille readers and are also only suitable to prototype haptic interaction as opposed to speech-based screen reader interaction. Also, the cardboard and plastic abstract models such as those used in [19] have a possible drawback of not allowing users to fully conceptualise the application as a whole, since users only interact with individual artefacts at a time.

In the following section, we describe our approach to engaging blind users in the design of a search interface. As well as including a blind user in the development team, we used a textual narrative scenario in a participatory design setting as a basis for dialogue to simulate interaction, in order to discuss design ideas with potential users.

\section{$3 \quad$ Using Scenarios for Participatory Design with Blind Users}

In this section, we describe the development of the proposed scenario-based approach. In Section 3.1, we explain the rationale for our overall approach to participatory design and in Section 3.2, we outline the steps included in developing the proposed scenario-based approach to verify requirements with blind users in the design of a search interface.

\subsection{Rationale for the Participatory Design Approach Taken}

To access the Web, blind users depend on a screen reader, a software application that by default reads web pages linearly from left to right, top to bottom, rendering the content in computer synthesised speech or Braille. Blind users also use the keyboard to navigate web pages and position the focus of the screen reader to read parts of the page of interest. Typical commands supported by screen readers include web page navigation forward/backward by headings (at different levels), forms, frames, edit fields, buttons and links. The linear rendition of text by screen readers plus the fact that they do not represent the spatial layout of web pages, such as columnised format, means that the mental models of blind users can vary significantly from those of sighted users [20].

There is a parallel to be drawn here between web navigation and navigation of real world spaces. Given due consideration, it is unlikely that when giving directions to a pedestrian, the way in which one would describe those directions would be the same for a sighted pedestrian as for a blind pedestrian. Instructions to the sighted pedestrian 
are likely to exploit visual cues, to be given at a granularity level appropriate to someone who can take in their surroundings at a glance. On the other hand, directions to a blind pedestrian, if they are to be useful, should be in terms of landmarks that are detectable by them, and at a level of granularity related to the way in which they interact with their surroundings, given whichever mobility aid they might employ, be it a dog or a white cane etc. Similarly, within human-computer interaction, in order to be useful, the way in which interactions are articulated need to take into account the senses and tools at the disposal of the user, as well as the level of granularity at which they interact with the system.

Based on this need to embed an understanding of how end users interact with the system at a deep level and the fact that other members of the development team can not easily share that experience (using a screen reader with a covered screen is not a realistic surrogate for a blind user with thousands of hours of screen reader experience [21]), it was decided that participatory design should be addressed at two levels.

Firstly, we included a blind user with knowledge of assistive technology as a full member of the design team. This provided the development team with immediate feedback in discussions about the development of appropriate inter- face artefacts, for example, properly labelled controls, the types of interactions supported by screen readers (the use of screen reader commands for web page navigation) and the appropriate vocabulary with which to describe interactions to blind users, for example, keystrokes rather than mouse clicks.

This understanding of how screen reader interaction works led to the development of a scenario and a dialogue about it being pitched at an appropriate level to make sense to a screen reader user. For example, the interface comprised several different components such as a search box, to which the user would frequently want to navigate. In this case, knowledge of screen reader interaction suggested that the appropriate way for this to be achieved should be through a keyboard shortcut and that an appropriate means of confirming that the action has been executed could be through playing a non-speech sound.

The second level at which participatory design was achieved was through the recruitment of 4 blind participants who took part in prototyping sessions to provide formative feedback to the design team. In these sessions, the overall scenario was used as the basis of dialogue about how users would interact with the system using a screen reader and the usefulness and usability of proposed interface features.

\subsection{Creating the Scenario-Based Approach}

During the requirements verification stage of the design process, the requirements of a system are analysed and validated to ensure that the designers and the users share the same understanding of the problems that were identified during the requirements gathering stage. For participatory design approaches, at this stage, designers communicate early design ideas to users to gather feedback [22].

In [23], we identified user requirements for a search interface for blind users through an observational study and in this paper, we verify requirements by using a scenario expressed as a textual narrative which then formed the basis of dialogue between the designers and the users. Basing this dialogue on a narrative scenario 
evoked a form of role play which worked well because the human mind is adept at overloading meaning in narrative structures [7, p. 54] which are meant to stimulate the imagination [9] and to provoke new ideas [10]. Therefore, they are well suited for use in participatory approaches to engage users early in design.

Our approach is a hybrid one involving a combination of participatory design [24] and the use of a detailed scenario to discuss ideas with target users [7]. The participation of a blind user as a member of the design team was invaluable when developing the scenario and its associated textual narrative as it helped us to conceptualise how potential users will interact with the system, given their use of screen readers. It also allowed us to establish the level of detail at which the scenario should be discussed with end-users. In Figure 1, we provide a broad overview of the framework we followed to implement the scenario-based approach and in the following we describe how we implemented each step of the framework in the design of a search interface for blind users. For each step, we also highlight the contributions of the blind member of the design team.

Proposed Interface Features

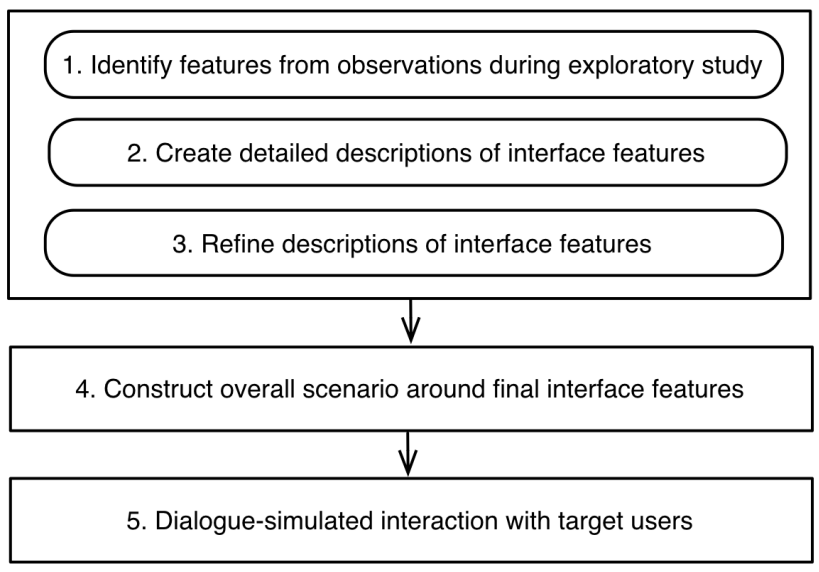

Fig. 1. Framework for the scenario-based approach

Step 1: Identify set of interface features. From our observations with blind users [23], we identified a set of search interface features that could support blind searchers during information seeking on the Web. These interface components were chosen to address the difficulties observed in [23] and were influenced by the design team's intuitions and knowledge of search user interface components. During this process, the blind team member contributed significantly from his knowledge and experience of using both graphical interfaces (via screen readers) and self-voicing auditory interfaces. This, to some extent, allowed the sighted designers to conceptualise the mental model they had to follow when designing interface components.

Step 2: Create detailed description of features. To communicate ideas for the search interface, we created detailed descriptions for all interface features. As we 
were using a textual narrative scenario, we had to ensure that our ideas were being conveyed correctly to the users and therefore, we focussed significantly on describing each interface component. For example, we proposed an integrated note-taking feature within the interface as described in the following:

Searchers can create a note and the system asks them where they would like to save this note and to give it a name. The note is divided in two parts: The first part of the note is editable by the searcher, that is, they can type ideas, copy and paste things from web pages etc. The second part cannot be edited and is used to save search results automatically by the system.

In this step, the contributions of the blind team member were significant in discussing the functionality of suggested support features as well as how searchers would interact with them. Through such discussions, we could ensure that interaction components were appropriate, and that the correct vocabulary was being used to describe interface components.

Step 3: Refine description iteratively. To ensure that the users shared the same understanding of the proposed features as the designers, we iteratively refined the description of the features through several informal conversations with the blind team member. For example, one idea was that a context menu might be useful to provide access to a set of options that become available when exploring individual search results. The idea of doing this through a context menu was contributed by the blind team member, who highlighted that context menus were familiar interaction artefacts to most screen reader users.

The blind team member further contributed that the way to initiate the interaction with end-users about the context menu should be by telling them to use a key combination (Shift+F10), rather than right clicking, as the keystroke is the usual way a blind user will initiate the interaction compared to the right mouse click familiar to sighted users. Therefore the blind team member gave us both an appropriate interaction artefact, and the most fitting means of describing the interaction to end- users. The options to be made available through the context menu were then identified and the best ways of implementing and describing the interactions to end-users were then refined through discussions between the sighted designers and the blind team member. The following was the final description used for the context menu:

You are aware that this new interface has a menu associated with each search result so that you can open, save, email and copy results. You hit the menu key and you find the following options in this particular order (Save Result, Copy, Email, Open). This is rather like the context menu you have in Windows that you bring up using Shift $+F 10$.

To enhance our textual description of some search support features, we referred to examples from other popular interfaces such as Google Search (results presentation 
with title, short description and web address) and Windows (context menu) that the users would be familiar with. These familiar points of reference helped the blind users to better envision the proposed search interface.

Step 4: Construct scenario around interface features. Once we finalised which search support features to include on the interface, we created an overall scenario, like a story, with a specific setting whereby the user was using a search interface for the first time after hearing about it from a friend. As we were also evaluating a new history mechanism and interface features to support searchers in resuming search tasks [25], the scenario included a stage where the user had to leave the task midway to attend an important appointment. When constructing the overall story, it was also essential to ensure the story included all suggested interface features in the correct order and in a reasonable sequence. For example, we would not describe a feature for managing search results before the users were asked to submit their first query.

Step 5: Dialogue-simulated interaction with the scenario. After the overall scenario was constructed, we used it as the basis of a formative evaluation with potential users (described in Section 4). The evaluator conducted a dialogue with each potential user to simulate the interaction with the interface components proposed in the scenario. At each step of the interaction, the evaluator would describe the interface feature to the user and explain how to interact with it. Then, the evaluator would ask the user for feedback on the feature and they would discuss the alternative interaction paths resulting from multiple design ideas.

\section{Evaluating the Use of Scenarios for Requirements Verification}

We evaluated the approach through a dialogue with potential users using the scenario to simulate interaction between the users and the yet-to-be constructed interface. Our goals for the evaluation were two-fold. Firstly, we wanted to verify the requirements for a new information-rich search interface for blind users, to communicate and discuss design ideas with potential users early in the design process. Secondly, our aim was to evaluate the use of scenarios in a participatory design setting for engaging blind users in the design process. Hence, in Section 5, we discuss the benefits, challenges and practical experiences of using the approach proposed in this paper.

\subsection{Participants}

We recruited 4 blind users through word of mouth and via online email lists. The participants were experienced searchers who rated their proficiency with assistive technologies from intermediate to advanced. Three of the participants were educated to a postgraduate level while one had professional qualifications in IT. In Table 1, we provide additional demographic information about these participants. 
Table 1. Demographics for all participants

\begin{tabular}{|l|l|}
\hline Age & 37 years \\
\hline Gender & M (3) F (1) \\
\hline Search Experience & 12 years \\
\hline Screen Reader & JAWS (3) VoiceOver (1) \\
\hline Frequency of Computer Use & Daily (3) Weekly (1) \\
\hline Use of Online Search Engine & Daily (3) Weekly (1) \\
\hline
\end{tabular}

\subsection{Procedure}

For each evaluation session, we used a standard script of the final scenario (described in step 4 of Section 3.2) to ensure that the users and ourselves shared the same understanding of the requirements for a new search interface. To begin with, the evaluator asked the user to think of a search task to complete. We left the choice of task open to elicit greater participation and user engagement with the scenario. The choice of search task did not affect the use of the script as it was built so that its primary focus was on interaction with individual interface components and thus could be adapted to any search task.

During the session, the evaluator who was the one in charge of the script, started the conversation with the participant by conducting a walk-through of the scenario in line with the script (The beginning of the dialogue-simulated interaction between the user and the evaluator is illustrated in Table 2). At each step, the evaluator provided the user with complete descriptions of the search interface feature and the user was prompted for feedback. The evaluator and the user also discussed how each interaction would work, including alternative interaction paths in case of multiple design ideas as shown in Table 3.

Table 2. Excerpt on query specification

Evaluator: Your friend has told you about a new search system and you would like to try it out for yourself to see how good it really is. Think of something you would like to search on this new system.

Once you have chosen your search task, you type the address of this new search inter- face in your web browser and you reach the page with the cursor in the search edit box.

"What do you type as a query?"

User: digital rights accessibility

Evaluator: You type this query and hit enter. If you misspell a word in your query, the system will specify which term you misspelt and allow you to submit a corrected version of your query. 


\subsection{User Feedback}

In this section, we describe the feedback gathered from 4 blind users during the evaluation of the scenario-based approach. The dialogue-simulated interaction between the designers and the users allowed us to gather feedback from users through their comments and critiques of suggested design plans. Additionally, we also use our own observations to categorise the user feedback as presented in the following:

Verifying Requirements. The scenario-based approach allowed us to verify requirements for a search interface for complex search tasks. We identified the requirements for a search interface during an observational study with 15 visually impaired searchers [23]. Through the proposed approach, we were able to ensure that the design team and the target users shared the same understanding of the difficulties faced by blind users when using current search interfaces.

In this respect, we were able to, for example, ascertain that spelling suggestions were a source of difficulties for searchers as the way misspelt words are rendered on current interfaces is not intuitive for screen reader users. One of the users said: "we hardly notice which term is misspelt. It would be good if the system clearly said which term is wrongly spelt".

This is because when spelling suggestions are presented to the user, the way in which the screen reader pronounces the suggestion for the correctly spelt word is often not detectably different from the pronunciation of the original misspelling, and so it is not clear what error is being corrected. In this case, the blind searcher can navigate to the suggestion and cursor character by character through it to find the difference, but this process loses all the immediacy of the visual representation. This was a difficulty that we had observed in [23].

Likewise, we were able to verify user requirements for a new history mechanism. In our scenario, we proposed a search history mechanism that would keep track of the queries submitted and the search results visited by the searcher. Participants in the evaluation commented on the need for such a history feature saying "I do not like the history in IE, this is more powerful than history. It allows you to call it up and instantly be back to where you were, in the same context" and "It is nice to pick up from where we left because sometimes we use keywords which are useful and then forget the right combination".

Identifying Issues with Current Design Ideas. By discussing design plans with potential users early in the design process, we were also able to identify issues with proposed design ideas from the users' perspective. Such discussions proved to be beneficial; for example, one of the design ideas for search results presentation included limiting the display of individual results to only one line per result on the search results page. Our reasoning for this idea was that it would reduce the amount of text that screen reader users would have to go through. However, we found that participants in the evaluation did not welcome this idea, as they would rather have some context about the search results retrieved by the search engine. They felt that if there was only one line per result, there would not be enough context to decide about the relevance of a specific result among those that the search engine had retrieved. 
During the dialogue-simulated interaction, the evaluator also had the opportunity to probe users on factors like keyboard navigation, which plays a central part in the user experience of blind searchers. Keyboard navigation is significantly different from visual navigation and hence, the design team have to ensure that all proposed interface features were intuitive to access via the keyboard.

In describing how they would interact with the interface, participants would often refer to how they would use the screen reader to access the proposed features. About the grouped approach for results presentation, one participant said "Along the lines of how VoiceOver works, this grouping on the page would be good" and another questioned how they would navigate back to a previous page "Would I need to use the screen reader key for this or would there be a special key combination?".

New Design Ideas Proposed by Users. Using the scenario-based approach allowed us to engage users in the development process via their interaction with a yet-to-be constructed search interface. Through this process, users came up with ideas of their own to enhance the design of some of the features that were being proposed. For example, in the scenario, we described a note-taking feature, which could be used by searchers to automatically save search results or to make notes of their own.

The initial idea was to allow users to then download or email the note in a text format. However, one of the participants highlighted that the benefits of having an integrated note facility could be enhanced by structuring the note and by including HTML tags to allow users to easily get back to any previously accessed web pages. About the same note-taking feature, another participant augmented our basic definition of the feature with his own design ideas, suggesting, "I can see where you are going with this, it could be in two panes, your browser and your search notes". The user was in fact proposing that there should be two separate areas on the interface, one for regular browser-related activities such as submitting queries and viewing web pages and the other area should be dedicated for note-taking and other search management activities. When users suggested such design ideas of their own, we discussed them with the design team including the blind co-designer to ensure that such an approach would be feasible and would enhance the users' experience.

\subsection{Discussion}

Engaging non-standard populations such as the elderly and disabled users in the design process is challenging as traditional user methodologies are not always effective at capturing the real user requirements. Therefore designers often have to explore different methods or adapt existing ones to ensure that such users can be successfully included in the design process [4][14].

In this paper, we described an approach, which included 2 levels of participatory design: we included a blind user in the design team and also carried out prototyping sessions with 4 blind users. Involving a blind team member who can combine a good knowledge of assistive technologies with an end-user perspective enabled us to create a scenario that was better matched to the vocabulary and interactions familiar to blind users. Thus, we could successfully engage blind users to solicit their feedback in the design of the search interface. 
Table 3. Excerpt on alternative search results presentation

Evaluator: There are alternative ways of presenting the search results retrieved:

1. Standard approach: Results are presented in a list with each result described using a title, a short summary and a web address. Each of these items is on a separate single line.

2. Simplified standard approach: Results are presented in a list, but each result is described in one line, with a title and a short summary.

3. New approach: Similar results are grouped together and you are presented with an overview of each group of search results. For example, results that deal with similar topics will be grouped together. If you are doing a travel task, web pages describing things to do at your destination will be grouped together and another group of pages could be about possible places to stay. If you would like to explore one of these groups, you can select the group and it will open in a different window and will contain all search results in that group described with title and a short summary. You can always return to the first window to browse through other result groups.

"What are your thoughts on these results presentation alternatives? Which one would you prefer and why?"

Search interfaces are highly interactive and to progress in their search task, searchers are required to perform activities such as formulate queries and view search results etc. The scenario-based approach described in this paper allowed us, to some extent, to simulate this interaction through a dialogue between the user and the designer. During the dialogue-based interaction, users were involved in the scenario and were constantly informed about their evolving interaction, for example, how search results are being handled and the alternative paths available to them. This approach to interaction elicited a high level of participation and engagement from the users, as evidenced by the feedback received. Therefore, such use of dialogue was beneficial as a model of engagement [26] and a model for effective communication and collaboration [27] between the designers and the potential users.

Overall, the findings gathered from the dialogue-simulated interaction showed that users had no problems in 'imagining' the interface proposed in the scenario [7]. The narrative was successful in evoking the search experience in users and therefore, they were able to discuss the proposed features for inclusion in the interface in the context of their use within the scenario, and to discuss alternative interaction sequences where they arose. The fact that users were able to go beyond the described interface features to question how they would interact on a relatively low level (screen reader keystroke level) is evidence that they were able to successfully form a mental model of the search interface that was yet-to-be constructed.

In addition, involving blind users at such an early stage allowed designers to identify limitations with their own design ideas. Participants would often question the practicality of the proposed interface features, requiring detailed explanations of how these interface components would be accessed in a realistically usable way with screen readers. Identifying these limitations at that stage ensured that no further 
development effort was put into interface features that would not meet the needs of the users, or would raise difficult usability issues.

The benefit of an inclusive approach, such as the one proposed in this paper, is that it enables users, especially those with disabilities, to become involved in the process of design and formative evaluation. This involvement in the development process encourages users to speak about their experiences with search interfaces and to contribute to design ideas and hence, the user truly becomes the centre of the design process. User-generated ideas during the scenario walk-through resulted in valuable contributions to our design plans. This is so because the participants in our study were experts at navigating the Web through screen readers and given their experience, they had better insights into how the overall search interface and the individual components would be perceived.

\section{Reflections on the Use of Scenarios for Participatory Design with Blind Users}

In this section, we reflect on the scenario-based approach and its evaluation with potential users. We discuss the benefits, challenges and practical experiences of using scenarios to engage blind users in the design process.

\subsection{Benefits}

Scenarios are flexible and adaptable and thus they can be customised according to the needs and abilities of the user group, for example, as a scenario-based drama for the elderly [12]. For our project, we created a textual narrative scenario for a dialoguebased interaction with blind users. The value of the scenario was that it allowed blind users to envision the proposed interface and form a mental model of how they would interact with it. This was important to correctly verify user requirements with blind users and also to rapidly communicate design ideas.

In addition, scenarios are adaptable in the level of detail that they convey to the user group, which can assist in enabling them to envision the proposed artefacts. For our approach, given our focus on requirements verification, we provided detailed descriptions for the proposed interface features and less detail about the interaction or the way certain tasks could be completed when using the search interface. For example, when describing a new search history mechanism, we fully described the items such as the queries and visited results that would be recorded as history, but we did not explicitly tell users how they would navigate the trail at a keystroke level. Instead, during the sessions, the users themselves wondered and discussed how they would interact with this history mechanism for different types of tasks.

In this way, we were able to achieve the comparable 'unfinished look' of handwritten mock-ups that Snyder [28] claims encourages creativity during low- fidelity paper prototyping. However, depending on the users' needs and the stage of the design process, a scenario-based approach could be used for more high-fidelity prototyping to evaluate how users would interact with the proposed artefacts. Our discussions with participants regarding how some interface features could be accessed through screen 
readers show that the use of scenarios is likely to be effective for such high fidelity prototyping.

In the absence of visual aids to communicate design ideas, sighted designers are likely to describe graphical user interfaces in a way that makes references to visual aspects of the interface, such as layout, structure etc. For the blind user, these descriptions would not be useful and would not convey a helpful representation of the interface features. For this reason, the involvement of a blind user was crucial to ensure that we used the right vocabulary and context to describe interactions at an appropriate level from the user's perspective.

Scenarios, especially when expressed as narratives, have an inherent ability to support participatory design [9][29] and thus complemented the level of participatory design reported in this paper. In such settings, scenarios furthered the communication between the users and the designers to enable successful collaboration [27]. We expressed the scenario in the form of a textual narrative (which was then used as the basis of a conversation between designers and users) and this enabled blind users to comment on the proposed design ideas in the context of screen reader access, as well as to suggest their own ideas for new or modified interface components.

\subsection{Challenges and Practical Experiences}

In the absence of visual aids, the designers in this approach relied on the textual descriptions of the interface features to communicate design ideas to the users. Therefore, the detailed descriptions played a significant role in shaping the mental model that users created of the interface. Using a standard script for the scenario ensured that variations in the way the interface was conceptualised were limited.

Our approach focussed entirely on the functionality of interface components and the way to interact with them. No efforts were directed towards conveying spatial information, which despite not necessarily being of primary importance to blind users, plays a role in how screen reader users perceive an interface, and very importantly, their collaborative use of the interface with sighted peers [20]. As an extension to this work, it will be interesting to examine the benefits and drawbacks of incorporating screen reader technology within the prototyping process, rather than the purely conversation-based approach taken here. It is unclear whether the incorporation of screen reader technology will enhance the realism of the interactions, and/or whether it may detract from the free flow of the dialogue about the interactions and their possible alternatives by overburdening the audio channel [30].

The approach proposed in this paper was a first attempt at using scenarios to engage blind users in the design process and hence, we identified some important points to consider for any future implementation or extension of this approach. Firstly, we expressed the scenario in a textual medium, with a dialogue-simulated interaction between the user and the designer. This audio-based approach works well with blind users, but as is common with audio interfaces, there is a lack of persistence. Therefore, any artefacts that are part of the scenario should be described in significant detail to ensure that users can conceptualise and "picture" the proposed design. Visual aids such as paper mock ups convey significant contextual information even in their most early versions and any attempt at replicating these types of approaches for blind users 
should be constructed using low-level details in the textual descriptions. Detailed descriptions can also be complemented with references to similar existing artefacts to convey as much contextual information as possible.

From the user evaluation, we concluded that scenarios, especially those expressed as a narrative, should be highly interactive to include the user as much as possible. Given that scenarios are stories about people and their activities, it is essential for users to feel part of the scenario to maximise their ability to envision the proposed interface. In the scenario, we regularly prompted users for feedback by asking them to think of a search task, by asking them for their query terms and by allowing them to choose the next step of their interaction etc. When scenarios are textual narratives and interaction with the user is dialogue-based, the designer will be speaking for relatively long periods to describe different parts of the interface. Therefore, to replicate an interactive search experience, users should be active 'actors' in the scenario activities to further user engagement.

Involving a blind person on the design team helped in many ways, but it is important to be aware of the dangers of over-relying on one person as a representative of a population. For example, the blind co-designer in our team was congenitally blind and had a lot of experience using JAWS with Windows and Internet Explorer to perform searches using Google, but only a passing knowledge of other screen readers, browsers and search engine combinations. Therefore, it is important to try to ensure relevant diversity [14], that is, users involved in the prototyping process, together with members of the design team, should provide a wide coverage of the range of tools and assistive technologies that might be used with the system being designed. It is also important to include users with less experience, as they will also be representative of members of the target population.

\section{Conclusion}

In this paper, we proposed a participatory approach based on a textual narrative scenario, tailored to the abilities of blind users to engage them in the design process. We evaluated the proposed approach with blind users and described the types of feedback we gathered in a participatory design setting through the use of a scenario as a basis for dialogue. The dialogue-simulated interaction between designers and users was effective in evoking the search experience in users and thus they could envision the yet-to-be constructed interface.

We achieved two levels of participatory design, namely by including a blind user in the design team and by carrying out prototyping sessions with 4 blind users. The contributions of the blind team member were invaluable to ensure that, in constructing the scenario, we used the right vocabulary and context to describe interactions at an appropriate level for screen reader users. In this paper, we also reflected on the benefits and challenges of our proposed approach and the practical experiences we gained in applying it so that it can be reused or further developed.

We believe that the proposed approach opens an interesting discussion on the ways to adapt current tools and techniques in user-centred design when designing for non-standard populations such as the elderly or users with disabilities. In this respect, 
future work could also focus on the comparison between different techniques to investigate the best ways of engaging users with disabilities in design depending on the stage of the design process and the type of feedback required.

\section{References}

1. Newell, A., Carmichael, A., Morgan, M., Dickinson, A.: The use of theatre in requirements gathering and usability studies. Interacting with Computers 18(5), 996-1011 (2006)

2. Clarkson, J.: Inclusive Design: Design for the Whole Population. Springer (2003)

3. Imrie, R., Hall, P.: Inclusive design: designing and developing accessible environments. Taylor \& Francis (2001)

4. Newell, A., Gregor, P.: User sensitive inclusive design - in search of a new paradigm. In: Proc. of the Conference on Universal Usability, pp. 39-44 (2000)

5. Newell, A., Gregor, P., Morgan, M., Pullin, G., Macaulay, C.: User-sensitive inclusive design. Universal Access in the Information Society 10(3), 235-243 (2010)

6. Gooda Sahib, N., Tombros, A., Stockman, T.: Evaluating a search interface for visually impaired users. Submission to the Journal of the American Society for Information Science and Technology (2013)

7. Carroll, J.M.: Making use: scenario-based design of human-computer interactions. The MIT Press (2000)

8. Rosson, M.B., Carroll, J.M.: Scenario-Based design. In: The Human-Computer Interaction Handbook: Fundamentals, Evolving Technologies and Emerging Applications, pp. 145-165. Taylor \& Francis (2007)

9. Jarke, M., Tung Bui, X., Carroll, J.M.: Scenario management: An interdisciplinary approach. Requirements Engineering 3(3), 155-173 (1998)

10. Bodker, S.: Scenarios in User-Centred design - setting the stage for reflection and action. In: Proc. of the Annual Hawaii International Conference on System, pp. 3053-3064 (1999)

11. Newell, A., Gregor, P.: Design for older and disabled people - where do we go from here? Universal Access in the Information Society 2(1), 3-7 (2002)

12. Marquis-Faulkes, F., McKenna, S., Gregor, P., Newell, A.: Scenario-based drama as a tool for investigating user requirements with application to home monitoring for elderly people. In: HCI International, Crete, pp. 512-516 (2003)

13. Bhatia, S., Dahn, C., Lee, J.C., Sampat, M., McCrickard, D.S.: VTAssist: a location-based feedback notification system for the disabled. In: Proc. of the Annual Southeast Regional Conference, pp. 512-517 (2006)

14. Lindsay, S., Jackson, D., Schofield, G., Olivier, P.: Engaging older people using participatory design. In: Proc. of CHI, pp. 1199-1208 (2012)

15. Okamoto, M.: Possibility of participatory design. In: Proceedings of the International Conference on Human Centered Design, pp. 888-893 (2009)

16. Metatla, O., Bryan-Kinns, N., Stockman, T., Martin, F.: Designing for collaborative crossmodal interaction. In: Proc. of Digital Futures (2011)

17. Brewster, S., Magnusson, C.: Guidelines for haptic Lo-Fi prototyping. In: Proc. of the NordiCHI, vol. 102, pp. 1483-1491 (2008)

18. Miao, M., Köhlmann, W., Schiewe, M., Weber, G.: Tactile paper prototyping with blind subjects. In: Altinsoy, M.E., Jekosch, U., Brewster, S. (eds.) HAID 2009. LNCS, vol. 5763, pp. 81-90. Springer, Heidelberg (2009)

19. Tanhua-Piiroinen, E., Raisamo, R.: Tangible models in prototyping and testing of haptic interfaces with visually impaired children. Guidelines for Haptic Lo-Fi Prototyping (2008) 
20. Stockman, T., Metatla, O.: The influence of Screen-Readers on web cognition. In: Proc. of the Accessible Design in the Digital World Conference (2008)

21. Petrie, H., Morley, S.: The use of non-speech sounds in non-visual interfaces to the MSWindows GUI for blind computer users. In: Proc. of the ICAD (1998)

22. Pekkola, S., Kaarilahti, N., Pohjola, P.: Towards formalised end-user participation in information systems development process. In: Proc. of the Conference on Participatory Design, pp. 21-30 (2006)

23. Gooda Sahib, N., Tombros, A., Stockman, T.: A Comparative Analysis of the Information Seeking Behaviour of Visually Impaired and Sighted Searchers. Journal of the American Society for Information Science and Technology 63(2), 377-391 (2012)

24. Muller, M.J.: Participatory design: The third space in HCI. In: Handbook of HCI 2nd edn., USA (2007)

25. Morris, D., Ringel Morris, M., Venolia, G.: SearchBar: a search-centric web history for task resumption and information re-finding. In: Proc. of CHI, pp. 1207-1216 (2008)

26. Wright, P., McCarthy, J.: Experience-centered Design. Morgan and Claypool Publishers (2010)

27. Anderson, R., Baxter, L.A., Cissna, K.N.: Dialogue: Theorizing Difference in Communication Studies. Sage Publications (2004)

28. Snyder, C.: Paper prototyping: the fast and easy way to design and refine user interfaces. Morgan Kaufmann (2003)

29. Luck, R.: Dialogue in participatory design. Design Studies 24(6), 523-535 (2003)

30. Chandrashekar, S., Stockman, T., Fels, D., Benedyk, R.: Using think aloud protocol with blind users. In: Proc. of ASSETS, pp. 251-252 (2006) 